\title{
Providing Support for Today's Decision Making Processes
}

\author{
S. Encheva \\ Stord/Haugesund University College \\ Bjørnsons g. 45, 5528 Haugesund, Norway
}

\begin{abstract}
Current decision making processes often involve uncertain information obtained from a number of sources. In addition the available data might be incomplete and/or collected at different time periods, i.e. its significance might require adjustments. In many cases experiments cannot be repeated and conclusions have to be drawn based on opinions of several experts. Working with intervals instead of using crisp values provides an opportunity to accommodate several varieties of data types. This can be done by involvement of grey numbers. Their exact values are unknown but either the corresponding intervals where they possibly belong are known or some sets of numbers where they can be placed are known. Grey numbers are further divided into conceptual, layer, and prediction type according to types of uncertainty they describe. In this work we consider project proposals ranking based on multi criteria multi person decision making. Grey numbers are employed to facilitate handling imprecise data. Concept lattices are further employed for querying in a set of formal concepts extracted from available data. Such processes result in establishing whether a new element belongs to any of the existing pattern structures.
\end{abstract}

Keywords- grey numbers; grey systems; evaluation; uncertainty

\section{INTRODUCTION}

Organizations and firms with reasonably small budgets usually have limited resources for undertaking new projects. Problems are getting even bigger when new proposals have to be considered and subsequently given priority. Decision support systems [10], [12] have been employed to handle selections and rankings in different areas. Some of them are based on methods taken from fuzzy operations, rough sets, soft sets and formal concept analysis, [2].

In research literature persons employed to evaluate attributes, objects, methods, etc. are usually referred to as experts. In some occasions they might very well be. In reality, however, such evaluations are often done by people with different backgrounds, experience, level of expertise, and positions in an organization. They might be in charge of finance, human resources, planning, etc. They have uneven influence on the final outcome and a wide range of preferences. This implies working with imprecise and incomplete data when f. ex. a decision support system is to be employed. Grey numbers and grey systems can handle humans' opinions expressed with uncertainty as well with non crisp values.

Ranking based on multi criteria multi person decision making is in the main focus of this work. Imprecise data is evaluated applying grey numbers.

\section{GREY NUMBERS AND THEIR APPLICATIONS}

In Grey theory is an effective method used to solve uncertainty problems with discrete data and incomplete information. The theory includes five major parts: grey prediction, grey relational analysis, grey decision, grey programming and grey control, [7], [8], and [11]. A quantitative approach for assessing the qualitative nature of organizational visions is presented in [16].

A grey number is generally denoted by " $\otimes ",[14]$, where a grey number with only a lower bound is denoted by $\otimes \in[a, \infty]$ or $\otimes(\underline{a})$; a grey number with only an upper bound is denoted by $\otimes \in[-\infty, \bar{a}]$ or $\otimes(\bar{a})$; and an interval grey number is denoted by $\otimes \in[\underline{a}, \bar{a}]$. There are also continuous and discrete grey numbers, black and white numbers, essential and nonessential grey numbers.

A grey system is defined as a system containing uncertain information presented by a grey number and grey variables, [13]. For an initial selection of items to be evaluated we propose use of formal concept analysis [6] and [9]. An information table is to be prepared first relating items and attributes. A concept lattice is to be depicted afterwards and with adequate use of association rules a selection of interesting items can be made. Later on expert's opinions for evaluating different aspects of each of the preselected items are to be gathered. They can be expressed by numerical and linguistic values. Converting linguistic values to numerical intervals can be done by f. ex. following [13], very poor [0, 1]; poor [1, 3]; mediu m poor $[3,4]$; fair $[4,5]$; mediu $\operatorname{mood}[5,6]$; $\operatorname{good}[6,9]$; very good $[9,10]$.

Addition or removal of experts' opinions is to be incorporated by applying operations with grey numbers, i.e. $\otimes_{1}+\otimes_{2} \in[a+c, b+d]$ and $\otimes_{1}-\bigotimes_{2}=\bigotimes_{1}+\left(-\bigotimes_{2}\right) \in[a-$ $d, b-c]$ where $\otimes_{1} \in[a, b], a<b$, and $\otimes_{2} \in[c, b], c<d$.

Suppose a decision group has $K$ persons, then the attribute weight of attribute $Q_{j}$ can be calculated as $\otimes w_{j}=$ $\frac{1}{K}\left[\otimes w_{j}^{1}+\otimes w_{j}^{2}+\cdots+\otimes w_{j}^{K}\right]$ where $\otimes w_{j}^{K}, j=1,2, \ldots, n$ is the attribute weight of $K$-th decision maker and can be described by grey number $\otimes w_{j}^{K}=\left[\underline{w}_{j}^{K}, \bar{w}_{j}^{K}\right]$. The rating values are $\otimes G_{i j}=\frac{1}{K}\left[\otimes G_{i j}^{1}+\otimes G_{i j}^{2}+\cdots+\otimes G_{i j}^{K}\right]$ where $\otimes G_{i j}^{K}, i=1,2, \ldots, m, j=1,2, \ldots, n$ is the attribute rating value 
of $K$-th decision maker and can be described by the grey number $\otimes G_{i j}^{K}=\left[\underline{G}_{i j}^{K}, \bar{G}_{i j}^{K}\right]$. The ideal alternative is considered to be the referential alternative. The grey possibility degree between tests in set $S=\left\{S_{1}, S_{2}, \ldots, S_{m}\right\}$ and ideal referential alternative $S^{\max }, P\left\{S_{i} \leq S^{\max }\right\}=\frac{1}{n} \sum_{1 \leq j \leq n} P\left\{\otimes V_{i j} \leq \otimes\right.$ $\left.G_{j}^{\max }\right\}$. A s maller $P\left\{S_{i} \leq S^{\max }\right\}$ implies worse ranking order of $S_{i}$.

The analytic hierarchy process [17] supports decision making where several factors are to be considered. Weight coefficients are attached to factors in order to distinguish their importance. Ranking problems have been previously considered by applying techniques from the analytic hierarchy process [17], [18] as well as mathematical programming methods [19].

Let $P$ be a non-empty ordered set. If $\sup \{x, y\}$ and $\inf \{x, y\}$ exist for all $x, y \in P$, then $P$ is called a lattice, [6]. In a lattice illustrating partial ordering of knowledge values, the logical conjunction is identified with the meet operation and the logical disjunction with the join operation. A context is a triple $(G, M, I)$ where $G$ and $M$ are sets and $I \subset G \times M$. The ele ments of $G$ and $M$ are called objects and attributes respectively, [20].

The rest of this section follows [4] where a query is a new object inserted in a formal context. A concept lattice defines a query space where each formal concept can be considered as a conjunctive Boolean query (i.e. a query where the constraint is given by the conjunction of the attributes in the intent of that formal concept) and a combination of formal concepts provides disjunction and negation. Various relevance measures such as topological distance within the lattice between the query concept and its superconcepts [4] semantic distance [1] extent intersection [15], and Jaccard similarity [3] have been already exploited.

\section{APPLICATIONS}

We consider six proposals $S 1, S 2, S 3, S 4, S 3$, and $S 6$ to be ranked with respect to three attributes: clarity of presentation $Q 1$, quality of proposed outcomes $Q 2$, and a degree to which a proposal supports firm's visions Q3. Linguistic ratings for attributes and weights are chosen by a committee. Attribute weights and ratings are considered as linguistic variables where $S=\left\{S_{1}, S_{2}, \ldots, S_{m}\right\}$ - a discrete set of $m$ possible alternatives, $Q=\left\{Q_{1}, Q_{2}, \ldots, Q_{n}\right\}$ - a set of $n$ attributes for assessment, $\otimes w=\left\{\otimes w_{1}, \otimes w_{2}, \ldots, \otimes w_{n}\right\}$ - the vector of attribute weights.

The calculations are done according to the theory presented in [14]. Linguistic ratings for attributes are performed first and calculations of attribute ratings are completed consequently. The weighted normalized decision table is shown in Table 1.
TABLE I. WEIGHTED NORMALIZED DECISION TABLE .

\begin{tabular}{|l|l|l|l|}
\hline Si & Q1 & Q2 & Q3 \\
\hline S1 & {$[0.47,0.95]$} & {$[0.26,0.46]$} & {$[4.5,5.5]$} \\
\hline S2 & {$[0.32,0.45]$} & {$[0.36,0.58]$} & {$[5.25,6.5]$} \\
\hline S3 & {$[0.43,0.55]$} & {$[0.28,0.50]$} & {$[3.0,4.25]$} \\
\hline S4 & {$[0.51,0.63]$} & {$[0.46,0.65]$} & {$[5.5,6.76]$} \\
\hline S5 & {$[0.31,0.52]$} & {$[0.27,0.56]$} & {$[4.78,6.1]$} \\
\hline S6 & {$[0.30,0.51]$} & {$[0.23,0.48]$} & {$[2.19,4.13]$} \\
\hline
\end{tabular}

The ideal referential alternative is $S^{\max }=\{[0.51,0.63],[0.36,0.65],[5.5,6.75]\}, \quad$ and the possibility degrees for $i=1,2,3,4$ are $\mathrm{P}\left\{\mathrm{S}_{\mathrm{i}} \backslash\right.$ leq $\left.S^{\max }\right\}=\{0.69$, $0.65,0.74,0.76,0.63,0.62\}$. The result of ranking is $S 4>$ $S 3>S 1>S 2>S 5>S 6$. According to the performed calculations the fourth proposal is the best choice.

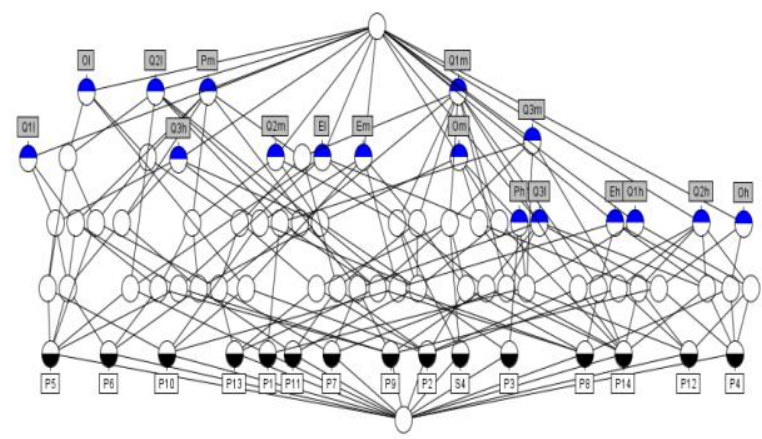

FIGURE I. A LATTICE WHERE THE QUERY IS S4.

More often than not differences between several proposals' rankings are marginal. Use query [4] from FCA for selecting a proposal satisfying 'requirements' not been part of the 'grey calculations'. Such requirements can be f. ex. the ones that are difficult to insert in the 'grey calculations'. Suppose there is data related to previous proposals including initial grading, final outcomes in terms of success and failure $O$, need for different expertise $E$, need for new partners $P$, need for additional financial support $F$, project management complications $M$, etc.

The remaining job is to find which concepts from the original context are related to the query concept and to what degree. At this point is important to consider seriously how to covert number intervals into linguistic values. Dividing f. ex. an interval $[0,1]$ into three subintervals of equal length according to three linguistic values 'low (l), medium (m), high 
(h)' might not always describe a practical case. Another suggestion can something like 'low - $[0,0.5]$ ', 'medium - [0.51, $0.75]$ ', and 'high - $[0.76,1]$ '.

TABLE II. DATA FROMPREVIOUS PROJECTS.

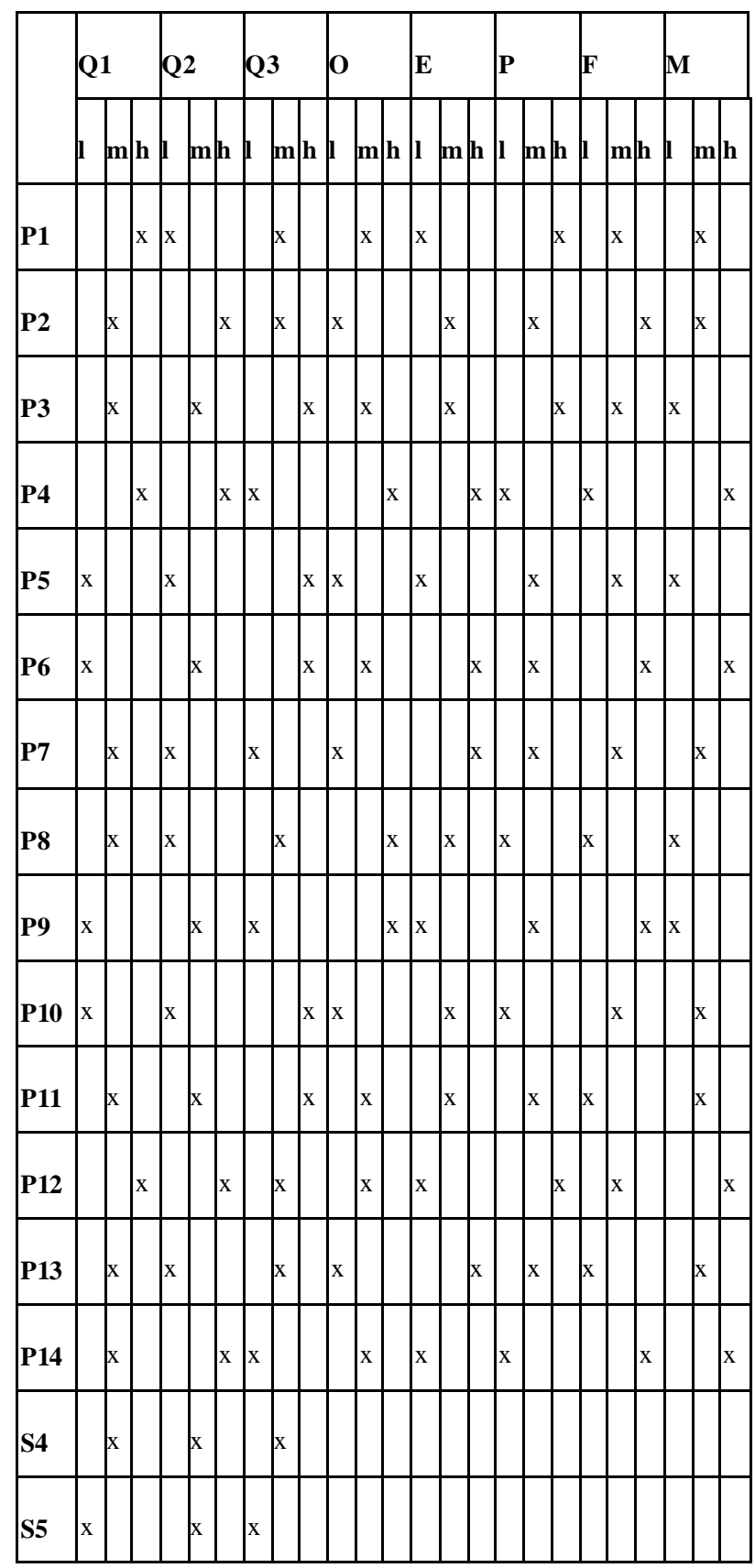

The lattice in Fig. 1 depicted from Table 2 has proposal S4 as a query. Attributes Q1 $\mathrm{m}$ and Q2m are shared by projects P3 and P11 and proposal S4, i. e. the the topological distance between $\mathrm{S} 4$ and $\mathrm{P} 3$ and $\mathrm{P} 11$ with respect to Q1 $\mathrm{m}$ and $\mathrm{Q} 2 \mathrm{~m}$ is zero. At the same time the topological distance between $\mathrm{S} 4$ and $\mathrm{P} 2, \mathrm{P} 8$ and $\mathrm{P} 13$ with respect to $\mathrm{Q} 1 \mathrm{~m}$ and $\mathrm{Q} 3 \mathrm{~m}$ is zero.
The lattice in Fig. 2 depicted from Table 2 has proposal S5 as a query. The topological distance between S5 and P9 with respect to Q11, Q2m and Q31 is zero. Attributes Q11 and Q2m are shared by projects P6 and P9 and proposal S5, while attribute Q31 is shared by projects $\mathrm{P} 4, \mathrm{P} 9, \mathrm{P} 14$ and proposal S5.

As emphasized in [1] distance measures based on clustering methods are often context-sensitive but they can handle important features which are outside of the reach of traditional statistical measures.

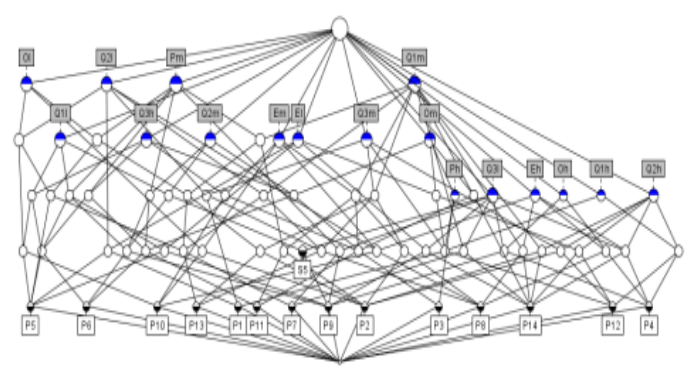

FIGURE II. A LATTICE WHERE THE QUERY IS S5.

Based on the available data one can say that proposal S5 is more likely to be in need of additional financial support than proposal S4, while proposal S4 is more likely to be in need of additional expertise than proposal S5. Such indications may affect the final decision on which proposal is to be granted.

As pointed in [1] it is worth investigating the potentials of concept lattices for dealing with ambiguous query terms in query refinement based on the actual content of the collection, [5].

\section{CONCLUSION}

While such an approach can definitely speed up selection processes it has to be kept in mind that different cases require some adjustments. Different organizations come usually with a variety of preferences and interests. All subjective views therefore ought to be incorporated in a decision support system serving a particular organization. Grey system theory is to be further employed for developing a Web based system collecting human opinions and ranking the preselected items.

\section{REFERENCES}

[1] Carpineto, C. \& Romano, G., Order theoretical ranking. Journal of the American Society for Information Science, 51(7), pp. 587-601, 2000.

[2] Carpineto, C. \& Romano, G., Concept Data Analysis: Theory and Applications, John Wiley and Sons, Ltd., 2004.

[3] Codocedo V., Lykourentzou I., \& Napoli A, Semantic querying of data guided by Formal Concept Analysis. In Formal Concept Analysis for Artificial Intelligence Workshop at ECAI 2012, 2012.

[4] Codocedo V., Lykourentzou I., Astudillo H., \& Napoli A., Using pattern structures to support information retrieval with Formal Concept Analysis, Proceedings of the International Workshop "What can FCA do for Artificial Intelligence?" Beijing, China, August 5, pp. 15-24, 2013.

[5] Cooper, J. \& Byrd, R., Lexical navigation: visually prompted query expansion and refinement, Proceedings of the Second ACM Digital Library Conference, Philadelphia, ACM, pp. 237-246, 1997. 
[6] Davey, B. A., \& Priestley, H. A., Introduction to lattices and order, Cambridge University Press, Cambridge, 2005.

[7] Deng J.L., Control problems of grey systems, System and control letters, 5, pp. 288-294, 1982.

[8] Deng, J.L., Introduction to grey system theory, Journal of grey systems, 1, pp. 1-24, 1989.

[9] Ganter B. \& Wille R.., Formal Concept Analysis, Springer, 1999.

[10] Gettinger J., Kiesling E., Stummer C., \& Vetschera R., A comparison of representations for discrete multi-criteria decision problems, Decision Support System,s 54, pp. 976-985, 2013.

[11] Hu Y.C., Grey relational analysis and radical basis function net work for determining costs in learning sequences, Applied mathematics and computation, 184, pp. 291-299, 2007.

[12] Huysmans J., Dejaeger K., Mues C., Vanthienen J., \& Baesens B., An empirical evaluation of the comprehensibility of decision table, tree and rule based predictive models, Decision Support Systems, 51, pp. 141$154,2011$.

[13] Li G.D., Daisuke Yamaguchi D., \& Nagai, M., A grey-based decisionmaking approach to the supplier selection problem, Mathematical and Computer Modelling, 46, pp. 573-581, 2007.

[14] Liu, S.Y., \& Lin Y., Grey Information Theory and Practical Applications, Advanced Information and Knowledge Processing, Springer, 2006

[15] Messai N, Devignes, M., Napoli A., \& Smail-Tabbone M., Using Domain Knowledge to Guide Lattice-based Complex Data Exploration. In Proceedings of the 2010 conference on ECAI 2010: 19th European Conference on Artificial Intelligence, pp. 847-852, 2010.

[16] Rahimnia F., Moghadasian M. \& Mashregh E., Application of grey theory organizational approach to evaluation of organizational vision, Grey Systems: Theory and Application, 1, 1, pp. 33-46, 2011.

[17] Saaty T. L. \& Peniwati K., Group Decision Making: Drawing out and Reconciling Differences. Pittsburgh, Pennsylvania: RWS Publications. 2008.

[18] Saaty T. L., Principia Mathematica Decernendi: Mathematical Principles of Decision Making, Pittsburgh, Pennsylvania: RWS Publications, 2010

[19] Smytka D.L. \& Clemens M. W., Total cost supplier selection problem: a case study, International Journal of purchasing and material management, 29, 1, pp. 42-49, 1993.

[20] Wille, R., Concept lattices and conceptual knowledge systems, Computers Math. Applications, 23(6-9), pp. 493-515, 1992. 